\title{
Исследование генерации СВЧ сигналов в планарном диоде Ганна с учетом радиационного облучения
}

\author{
(C) Е.С. Оболенская ${ }^{+}$, Е.А. Тарасова ${ }^{+}$, А.Ю. Чурин ${ }^{+}$, С.В. Оболенский ${ }^{+}$, В.А. Козлов* \\ + Нижегородский государственный университет им. Н.И. Лобачевского, \\ 603950 Нижний Новгород, Россия \\ * Институт фризики микроструктур Российской академии наук, \\ 603087 Нижний Новгород, Россия \\ E-mail: bess009@mail.ru, obolensk@rf.unn.ru
}

(Получена 27 апреля 2016 г. Принята к печати 10 мая 2016 г.)

Теоретически исследована генерация СВЧ сигнала в планарных диодах Ганна с двумерным электронным газом, в которых ранее нами исследовался стационарный транспорт электронов. Рассмотрены возможности использования управляющего электрода, аналогичного затвору полевого транзистора, для управления параметрами выходного СВЧ сигнала диода. Проведено сравнение результатов физико-топологического моделирования для полупроводниковых структур с различной конструкцией активной области диодов без квантовой ямы, с одной и двумя квантовыми ямами, разделенными потенциальным барьером. Результаты расчетов сопоставлены с полученными нами ранее экспериментальными данными по регистрации ганновской генерации в полевом транзисторе с затвором Шоттки. Теоретически и экспериментально показано, что мощность сигнала, генерируемого планарным диодом Ганна с квантовой ямой и управляющим электродом, достаточна для реализации монолитных интегральных схем (МИС) различного функционального назначения. Теоретически и экспериментально показано, что благодаря использованию управляющего электрода за счет введения корректирующей обратной связи возможно значительное увеличение радиационной стойкости СВЧ генератора полевых транзисторов с затвором Шоттки.

\section{1. Введение}

Дельта-легированные структуры с квантовыми ямами [1], содержащими двумерный электронный газ, находят широкое применение в современных планарных диодах Ганна. Ранее нами было показано [2], что высокое значение отрицательной дифференциальной проводимости (ОДП), реализуемое в подобных структурах, позволяет повысить предельные частоты и мощность планарных диодов Ганна в несколько раз. Кроме того указанная в [2] конструкция позволяла создавать радиационностойкие диоды Ганна.

Важно, что использование планарной конструкции диода Ганна позволяет сочетать преимущество диодной технологии изготовления сверхвысокочастотных (СВЧ) генераторов с возможностями дальнейшей обработки полезного сигнала с помощью полевых транзисторов Шоттки, изготовленных на одном кристалле в рамках единой технологии создания монолитной интегральной схемы (МИС). Однако на основе транзисторов могут быть также реализованы СВЧ генераторы за счет обратной связи, полученной обычными радиотехническими методами. В связи с этим необходимо сопоставить параметры указанных типов генераторов и указать те области применения, где различные типы (транзисторный и диодный) будут иметь свои преимущества. Указанное приобретает особую важность при создании радиационно-стойких СВЧ генераторов, когда возможности компенсации ухода параметров генератора за счет изменения электрических режимов питания и управления позволяют нивелировать ухудшение свойств прибора из-за радиационного воздействия.
Так, в [3] было показано, что транзисторные СВЧ генераторы, управляемые напряжением (ГУН), без специальных мер схемотехнической компенсации имеют низкую радиационную стойкость из-за ухода частоты генерации после радиационного облучения. Напротив, применение цепочек автокоррекции в цепи затвора позволяло компенсировать как изменения, связанные с радиационным воздействием, так и температурный дрейф параметров генератора. Недостатком такого подхода является относительная сложность проектирования, связанная с тем, что на затвор транзисторов приходится подавать как постоянное компенсирующее смещение различного уровня, так и переменный сигнал через цепь обратной связи. Изменение постоянного смещения затвора приводит к изменению параметров транзистора даже без нагрева и радиационного воздействия. Поэтому определить алгоритм компенсации указанных дестабилизирующих факторов оказывается достаточно сложно - требуются экспериментальные исследования для определения характерных констант изменения параметров генератора при облучении и нагреве. Все это существенно сказывается на времени выполнения работы и ее стоимости. Использование управляющих контактов в диодах Ганна позволяет разрешить указанное противоречие, так как на них подается только постоянное смещение, т.е. процедура проектирования генератора будет облегчена, если появится информация о возможностях такого управления.

Ранее в [4] экспериментально и теоретически была показана возможность возбуждения ганновской генерации в полевых транзисторах Шоттки и было указано, что электрод затвора может играть роль управляющего электрода в диоде Ганна. Также в [2,5-7] обсуждалась 
радиационная стойкость как диодов Ганна, так и полевых транзисторов, в том числе с квантовой ямой (high electron mobility transistor, HЕМТ). В данной работе проведено сравнение новых данных с полученными ранее и обсуждаются особенности ганновской генерации в диодах с управляющим электродом, с одной или двумя классическими и квантовыми ямами, разделенными потенциальным барьером. Кроме того, увеличение проводимости канала транзисторов за счет использования нескольких параллельно включенных квантовых ям [8] позволяет повысить его выходную мощность и улучшить иные СВЧ параметры. Поэтому здесь нами были исследованы возможности улучшения аналогичных параметров диодов Ганна.

\section{2. Конструкция и технология изготовления исследуемых диодов}

Для проведения моделирования ганновской генерации в планарных диодах с управляющим электродом для структур с двумерным газом и обычным (классическим) проводящим слоем полупроводника (рис. 1) использовался физико-топологический подход, т.е. в качестве исходных данных задавались параметры слоев полупроводниковой структуры и анализировались особенности транспорта электронов, формирования доменов Ганна и влияния на эти процессы радиационного воздействия.

Контакты анода и катода исследуемых диодов изготавливались по традиционной технологии на основе композиции $\mathrm{Au}-\mathrm{AuGe}$. B случаe GaAs-структур верхняя часть слоя 1 (рис. 1) легировалась до уровня $5 \cdot 10^{18} \mathrm{~cm}^{-3}$ и проводилось вжигание контактов, так что их контактное сопротивление становилось незначительным [4]. В случае использования структур на основе InGaAs металлические контакты располагались на специальном $\delta$-легированном слое, снижающем сопротивление контактов не столь значительно [2]. Использовалась конструкция управляющего электрода на основе барьера Шоттки, которая во всех случаях была такой, что позволяла надежно изолировать электрод и снизить его токи утечки до значений, сопоставимых с аналогичными в полевых транзисторах Шоттки и НЕМТ [4-6]. В частности, использовалась технология подтравливания верхней части слоя 1 так, чтобы основание управляющего электрода легло на нижнюю часть слоя 1 с относительно низкой проводимостью [3].

Исследовались несколько вариантов конструкции диодов. В диодах с одним каналом слои 3 и 4 не применялись, а квантовая яма формировалась с помощью слоя InGaAs толщиной 12 нм или отсутствовала, если использовался только легированный слой GaAs. В диодах c двумя каналами как классические, так и квантовые ямы формировались аналогично, а разделительный слой 3 из слабо легированного $\mathrm{GaAs}$ толщиной 20 нм позволял обмениваться носителями заряда между ямами. Концентрации электронов в указанных каналах составляли

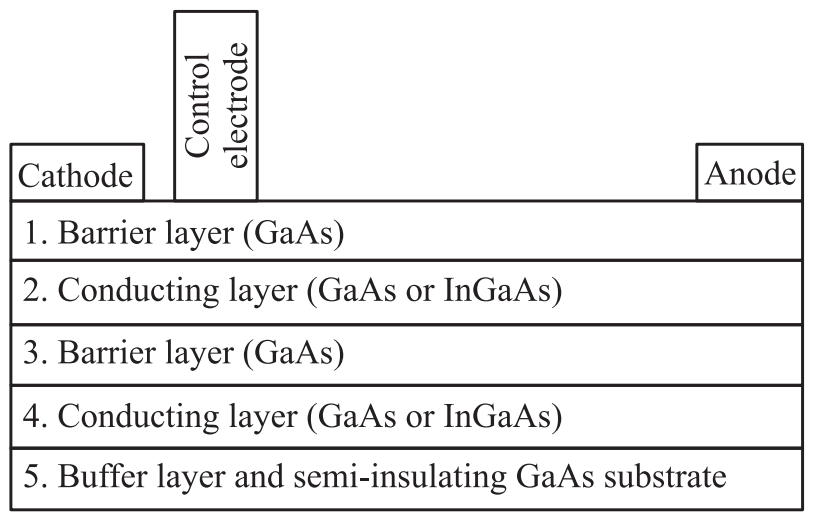

Рис. 1. Схематическое изображение исследуемых структур. Длина управляющего электрода 0.25 мкм.

$5 \cdot 10^{12} \mathrm{~cm}^{-2}$, что коррелировало с ранее рассмотренными конструкциями диодов и транзисторов [2-7] и позволяло калибровать применяемую для расчетов модель по экспериментальным данным, указанным в этих работах, с целью корректного сопоставления новых данных с полученными ранее.

\section{3. Особенности компьютерной модели и методики расчета}

Для расчетов использована двумерная численная модель в квазигидродинамическом приближении [2]. Самосогласованно решалась нестационарная система уравнений Пуассона, непрерывности, баланса энергии и импульса, дополненная выражениями для тока и потока энергии электронов. Так как сопротивления металлических контактов, особенно в структурах с квантовыми ямами, давали существенный вклад в параметры диодов, учет указанных параметров проводился на основе экспериментальных данных, что позволяло калибровать модель таким образом, чтобы теория и эксперимент хорошо коррелировали.

Суть метода калибровки состояла в следующем. В рамках одного теоретического подхода сопоставлялись вольт-амперные и СВЧ характеристики диодов с управляющим электродом, имеющие один классический канал (два канала) или канал на основе кватовой ямы (двух квантовых ям). Параметры контактных сопротивлений учитывались аналитически, так что вычисление напряженности электрического поля в активной области структуры становилось корректным [2]. Последнее позволяло адекватно сопоставлять результаты расчетов и экспериментов.

После сопоставления результатов расчета статических и импульсных вольт-амперных характеристик с экспериментальными данными и настройки модели удовлетворительным образом проводилось моделирование процессов генерации в исследуемых диодах. При этом соответствие результатов моделирования и экспериментальных данных для случая одиночного классического 
и квантового каналов с погрешностью не более $15 \%$ позволяло говорить об адекватности моделирования и для случая двух квантовых каналов.

Влияние облучения быстрыми нейтронами спектра деления со средней энергией 1 МэВ учитывалось в модели путем изменения концентрации и скорости носителей заряда из-за введения точечных радиационных дефектов и их кластеров при нейтронном облучении [2,5-7]. Кроме того, вводилось изменение времен релаксации энергии и импульса электронов согласно данным [2,5-7], что позволяло детально учесть процессы разогрева электронного газа. Полученные в ходе расчетов изменения параметров высокочастотного сигнала, генерируемого диодами, сравнивались с экспериментальными данными. Определялась возможность компенсации изменения параметров сигнала путем изменения постоянных напряжений питания и управления. Абсолютные значения рассеиваемой мощности диодов достигали $10 \mathrm{MBT}$, поэтому для расчетов их температуры использовалась информация о конструкции теплоотвода из [2], а также данные импульсных измерений, позволившие определить температуру экспериментально. Рассчитанная температура рабочей области диодов учитывалась при проведении моделирования транспорта электронов.

\section{4. Расчеты и эксперименты}

На рис. 2 проводится сравнение нормированных вольт-амперных характеристик (BАХ) исследованных диодов Ганна различной конструкции. Сопоставление с экспериментом показало, что погрешность расчета ВАХ для диодов с одной классической или одной квантовой ямой не превышает 15\%. Поскольку одна и та же модель применялась для моделирования всех диодов, указанная погрешность, по-видимому, будет соответствовать диодам с двумя квантовыми ямами.

Как видно из рис. 2, отрицательная дифференциальная проводимость диодов существенно зависит от типа и

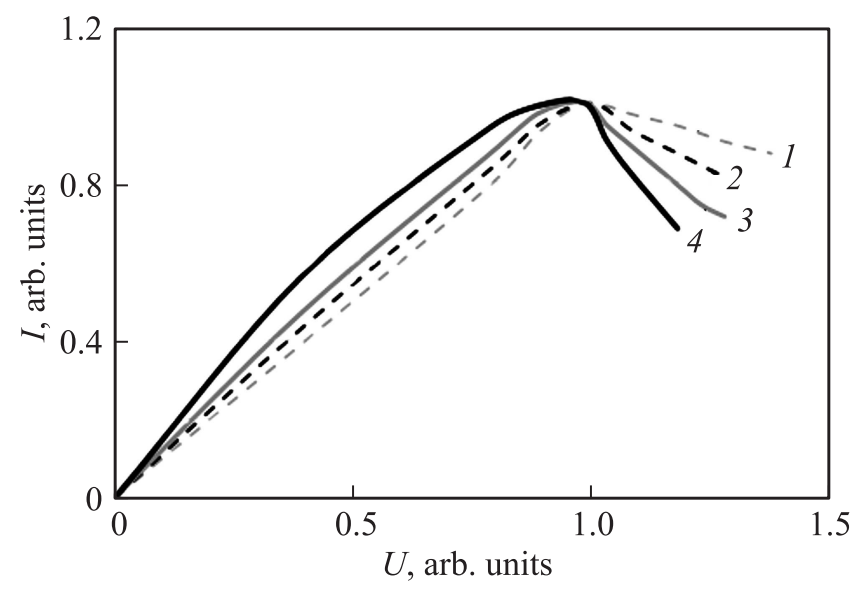

Pис. 2. Нормированные на значение тока и напряжение в максимуме расчетные ВАХ диодов: 1 - одна классическая яма, 2 - одна квантовая яма, 3 - две классические ямы, 4 - две квантовые ямы.
Таблица 1. Изменение величины отрицательной дифференциальной проводимости диодов (\%) после нейтронного облучения с различным флюенсом

\begin{tabular}{|c|c|c|c|}
\hline \multirow{2}{*}{ Тип диода } & \multicolumn{3}{|c|}{ Флюенс, см $^{-2}$} \\
\hline & $1 \cdot 10^{14} \mathrm{~cm}^{-2}$ & $2 \cdot 10^{14} \mathrm{~cm}^{-2}$ & $5 \cdot 10^{14} \mathrm{~cm}^{-2}$ \\
\hline 1 классическая яма & $-6(0)$ & $-15(-7)$ & $-35(-15)$ \\
\hline 1 квантовая яма & $-5(0)$ & -9 & $-23(-10)$ \\
\hline 2 классические ямы & $-6(0)$ & $-12(-4)$ & $-17(-5)$ \\
\hline 2 квантовые ямы & $-3(0)$ & $\begin{array}{ll}-6 & (0)\end{array}$ & $-14 \quad(0)$ \\
\hline
\end{tabular}

Примечание. В скобках указаны возможности компенсации влияния радиационного облучения за счет изменения напряжения на управляющем электроде.

количества ям. Квантовые ямы выигрывали за счет бо́льших (в 1.2-1.5 раза) подвижности и средней скорости электронов. Кроме того, увеличение количества ям также дает выигрыш в повышении отрицательной дифференциальной проводимости.

Важным результатом экспериментов и расчетов была демонстрация возможностей управления величиной „ганновской“ ОДП путем изменения напряжения на управляющем электроде. При увеличении отрицательного потенциала величина ОДП снижалась до нуля и по виду ВАХ становилась аналогичной характеристике полевого транзистора Шоттки. При подаче положительного потенциала на управляющий электрод величина ОДП также падала, что объясняется отпиранием барьера Шоттки и соответственно резким увеличением тока управляющего электрода. Изменение толщины слоя 1 структуры (рис. 1), а также уровня легирования слоев 2 и 4 позволяло смещать значение напряжения управляющего электрода, соответствующего максимуму значения ОДП, что коррелирует с данными [4].

В табл. 1 приведены результаты моделирования влияния радиационного облучения быстрыми нейтронами спектра деления со средней энергией 1 МэВ на величину отрицательной дифференциальной проводимости диодов. Пороговый флюенс составлял для всех типов диодов $(3-5) \cdot 10^{13} \mathrm{~cm}^{-2}$, а скорость снижения указанного сопротивления была различна: диоды с квантовыми ямами меньше чувствовали радиационное облучение. Последнее объясняется меньшей чувствительностью скорости электронов в квантовых ямах к радиационному воздействию. Это коррелирует с данными работ [5-7].

Путем изменения напряжения управления удавалось частично или даже полностью компенсировать радиационное воздействие. Величина снижения ОДП с учетом компенсации напряжением управления показана в табл. 1 в скобках. Следует отметить, что если установить исходное напряжение таким, чтобы ОДП была меньше максимального на 20-30\%, то после облучения возможно не только достижение полной компенсации, но и увеличение значения ОДП выше исходного путем его выведения в максимальное значение. Это открывает возможности для разработки специальной технологии, 
позволяющей повысить радиационную стойкость генераторов на основе указанных диодов.

Далее проводилось моделирование процесса генерации СВЧ сигнала, рассчитывались его амплитуда и частота. Сопоставление результатов расчета с экспериментальными данными, полученными для диодов с одной классической или квантовой ямой, показало, что погрешность расчетов составляет $15-25 \%$ и носит систематический характер. После калибровки модели удалось снизить погрешность до 5-15\%.

На рис. 3 показана зависимость амплитуды $A$ и частоты $F$ генерируемого сигнала от напряжения питания для диодов с одной квантовой ямой до и после облучения. Поведение диодов других конструкций (табл. 1) было аналогичным. Эффективность электронной перестройки частоты при изменении напряжения на аноде составляла $1-2$ ГГц/В, а при изменении напряжения на управляющем электроде $0.3-1$ ГГц/В. Максимальная мощность сигнала достигала $10 \mathrm{MB}$ на $1 \mathrm{mм}$ ширины затвора для диодов с двумя квантовыми ямами, а наименьшая мощность реализовывалась в диоде с одной классической ямой $-1.5 \mathrm{MBT} / \mathrm{Mм}$. Эти данные коррелируют с результатами экспериментальных исследований параметров СВЧ сигнала, генерируемого диодом Ганна с классическим каналом [4].

Предельные частоты генерации диодов определялись длиной пролетной области в соответствии с теорией классических диодов Ганна для длин > 1 мкм, а при снижении длины пролета до 0.5 мкм и менее наблюдалось расхождение с классической теорией, связанное с влиянием эффекта всплеска скорости (overshoot) [1,6]. Расчеты показали, что предельная частота генерации 350 ГГц будет соответствовать диоду с двумя квантовыми ямами и длиной пролетной области 300 нм. Этот результат коррелирует с данными работы [1].

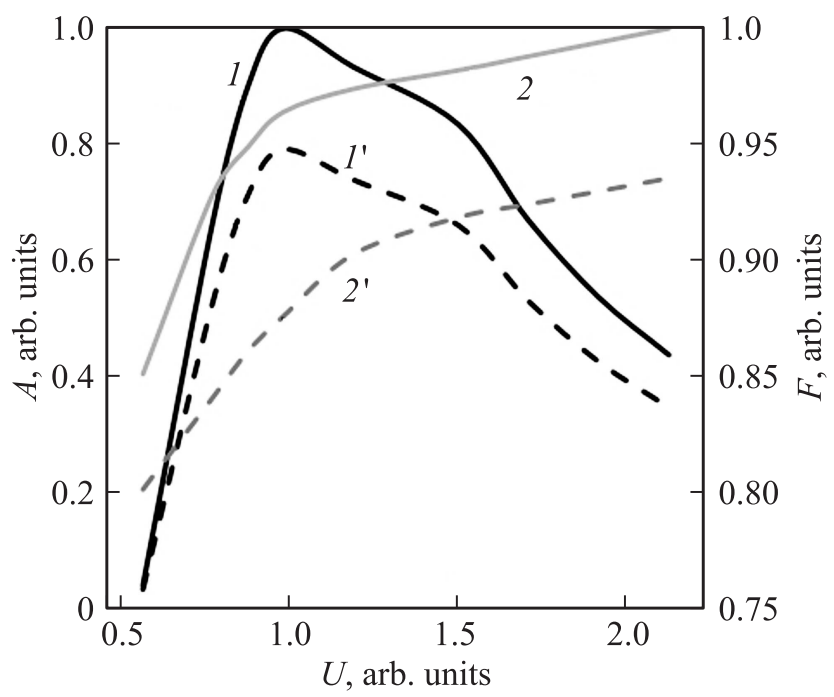

Рис. 3. Зависимость амплитуды $A\left(1,1^{\prime}\right)$ и частоты генерации $F\left(2,2^{\prime}\right)$ от напряжения питания $U$ до $(1,2)$ и после $\left(1^{\prime}, 2^{\prime}\right)$ нейтронного облучения спектра деления с флюенсом $1 \cdot 10^{14} \mathrm{~cm}^{-2}$.
Таблица 2. Результаты расчетов изменения амплитуды генерируемого сигнала после нейтронного облучения

\begin{tabular}{l|c|c|c}
\hline \multirow{2}{*}{\multicolumn{1}{c|}{ Тип диода }} & \multicolumn{3}{|c}{ Флюенс, $\mathrm{cm}^{-2}$} \\
\cline { 2 - 4 } & $1 \cdot 10^{14} \mathrm{~cm}^{-2}$ & $2 \cdot 10^{14} \mathrm{~cm}^{-2}$ & $5 \cdot 10^{14} \mathrm{~cm}^{-2}$ \\
\hline 1 классическая яма & -15 & -39 & -90 \\
1 квантовая яма & -13 & -29 & -54 \\
2 классических ямы & -18 & -34 & -65 \\
2 квантовых ямы & -9 & -17 & -41
\end{tabular}

Моделирование изменения параметров диодов при нейтронном облучении показало, что наиболее стойкими диодами являются приборы, имеющие две квантовые ямы (табл. 2). Для них были зарегистрированы в 2-3 раза бо́льшие пороговые значения уровней облучения, при которых уменьшение мощности выходного сигнала составляет $10 \%$. Это объясняется более высокими значениями концентрации электронов в проводящих слоях, так что при фиксированном уровне радиационных дефектов относительные изменения концентрации электронов меныше.

Как следует из результатов расчетов и экспериментов, благодаря наличию у диодов дополнительного управляющего электрода возможна коррекция частоты генерируемого сигнала, которая изменяется из-за облучения за счет повышения частоты рассеяния на радиационных дефектах и снижения скорости электронов. Результаты показывают, что возможна полная компенсация ухода как частоты генерации, так и амплитуды выходного сигнала диодов с двумя квантовыми ямами при флюенсах облучения $<1.5 \cdot 10^{14} \mathrm{~cm}^{-2}$. Для других диодов аналогичное значение флюенса колеблется от 0.8 до $1.4 \cdot 10^{14} \mathrm{~cm}^{-2}$.

При бо́льших флюенсах возможна лишь частичная компенсация либо полная компенсация одного из указанных параметров в ущерб сопряженному другому. Поскольку речь идет об управлении параметрами выходного сигнала генератора за счет изменения постоянных напряжений управления и питания, то схемотехническая реализация цепочек указанного смещения, в том числе и автосмещения, позволит создавать МИС по более простому технологическому маршруту, чем в случае использования классических транзисторных генераторов.

\section{5. Заключение}

Результаты расчетов и экспериментов показывают, что возможно создание планарного диода Ганна на структурах с двумя квантовыми ямами и управляющим электродом Шоттки, позволяющим эффективно компенсировать уход параметров выходного сигнала, возникающий из-за радиационного облучения.

Расчеты и эксперименты показали, что для всех типов диодов пороговый флюенс составлял $(3-5) \cdot 10^{13} \mathrm{~cm}^{-2}$, 
а существенные изменения ВАХ, амплитуды выходного сигнала и его частоты происходили при флюенсах $(2-5) \cdot 10^{14} \mathrm{~cm}^{-2}$. Изменениями напряжения на управляющем электроде и в цепи питания удается провести полную компенсацию изменения параметров сигнала вплоть до флюенсов $1.5 \cdot 10^{14} \mathrm{~cm}^{-2}$ в случае диодов с двумя квантовыми ямами. Для других диодов аналогичное значение флюенса колеблется от $0.8 \cdot 10^{14}$ до $1.4 \cdot 10^{14} \mathrm{~cm}^{-2}$.

Итак, полученные в работе данные свидетельствуют о возможности реализации МИС с электрической компенсацией изменения параметров диодов, произошедших из-за процессов радиационной деградации.

Работа выполнена при поддержке грантов РФФИ (№ 14-02-0058, 15-02-07935), МОН РФ (соглашение от 27.09.2013 г. № 02.В.49.21.003) и Государственным заданием МОН РФ (код проекта 2183).

\section{Список литературы}

[1] A. Khalid, N.J. Pilgrim, G.M. Dunn et al. IEEE Electron. Dev. Lett., 28 (10), 849 (2007).

[2] Е.С. Оболенская, Е.А. Тарасова, А.Ю. Чурин, С.В. Оболенский, В.И. Шашкин, А.В. Мурель. ФТП, 29 (11), 1507 (2015).

[3] Д.И. Дюков, А.В. Баев, А.Н. Качемцев и др. $T p . X V$ omраслевого координационного семинара по СВЧ технике (Нижний Новгород, Россия, 2007) с. 79.

[4] М.А. Китаев, С.В. Оболенский. Микроэлектроника, 3, 1 (2001).

[5] Е.А. Тарасова, Д.С. Демидова, С.В. Оболенский, А.Г. Фефелов, Д.И. Дюков. ФТП, 46 (12), 1587 (2012).

[6] С.В. Оболенский. Изв. вузов. Электроника, 6, 31 (2003).

[7] Е.А. Тарасова, А.В. Хананова, С.В. Оболенский, В.Е. Земляков, Ю.Н. Свешников, В.И. Егоркин, В.А. Иванов, Г.В. Медведев, Д.С. Смотрин. ФТП, 50 (3), 331 (2016).

[8] М. Шур. Перспективные приборы на основе GaAs (М., Мир, 1992).

Редактор Л.В. Шаронова

\section{Research of microwave signals generation in planar Gunn diodes in view of radiation exposure}

\section{E.S. Obolenskaya ${ }^{+}$, E.A. Tarasova ${ }^{+}$, A.Yu. Churin ${ }^{+}$,} S.V. Obolensky+, V.A. Kozlov*

\author{
+ Lobachevsky State University of Nizhni Novgorod, \\ 603950 Nizhny Novgorod, Russia \\ * Institut for Physics of Microstructures, \\ Russian Academy of Sciences, \\ 603087 Nizhny Novgorod, Russia
}

\begin{abstract}
The microwave signal generation in planar Gunn diodes with two-dimensional electron gas in which we have previously studied a stationary electron transport was investigated theoretically. The possibilities of using the gate electrode, analogical to the gate of field-effect transistor, to control the parameters of the microwave signal output diode were considered. A comparison of the results of physical and topological modeling of semiconductor structures with different active area diode structure - without the quantum well, with one and two quantum wells separated by a potential barrier, was done. The calculation results are compared with our earlier experimental data on the registration Gunn generation in the field-effect transistor with the Schottky gate. The power of the signal generated by the planar Gunn diode with a quantum well and a gate electrode, is sufficient for the implementation of MIS for various applications were shown theoretically and experimentally. It is shown theoretically and experimentally that by using the gate electrode, through the implementation of corrective feedback may significantly increase the radiation resistance of the microwave generator.
\end{abstract}

